\title{
We are living in the geologic age when human activities have dramatically affected our planet and its environment
}

\section{Michele Barry MD FACP \\ Founder, WomenLift Health \\ Senior Associate Dean and Director, Center for Innovation in Global Health, Stanford University}

\section{Gail A. Reed MS}

Dr Barry is incoming Board Chair of the Consortium of Universities for Global Health (CUGH) and past President of the American Society of Tropical Medicine and Hygiene. She is a member of the National Academies of Sciences, Engineering, and Medicine, and serves on the National Academy of Medicine's Board of Global Health. She has received the Elizabeth Blackwell Medal for outstanding contributions to women in the field of medicine, and is founder of WomenLift Health, a global initiative to transform global health outcomes by unleashing and elevating talented mid-career women to become global health leaders.

Michele Barry (r.) with women leaders

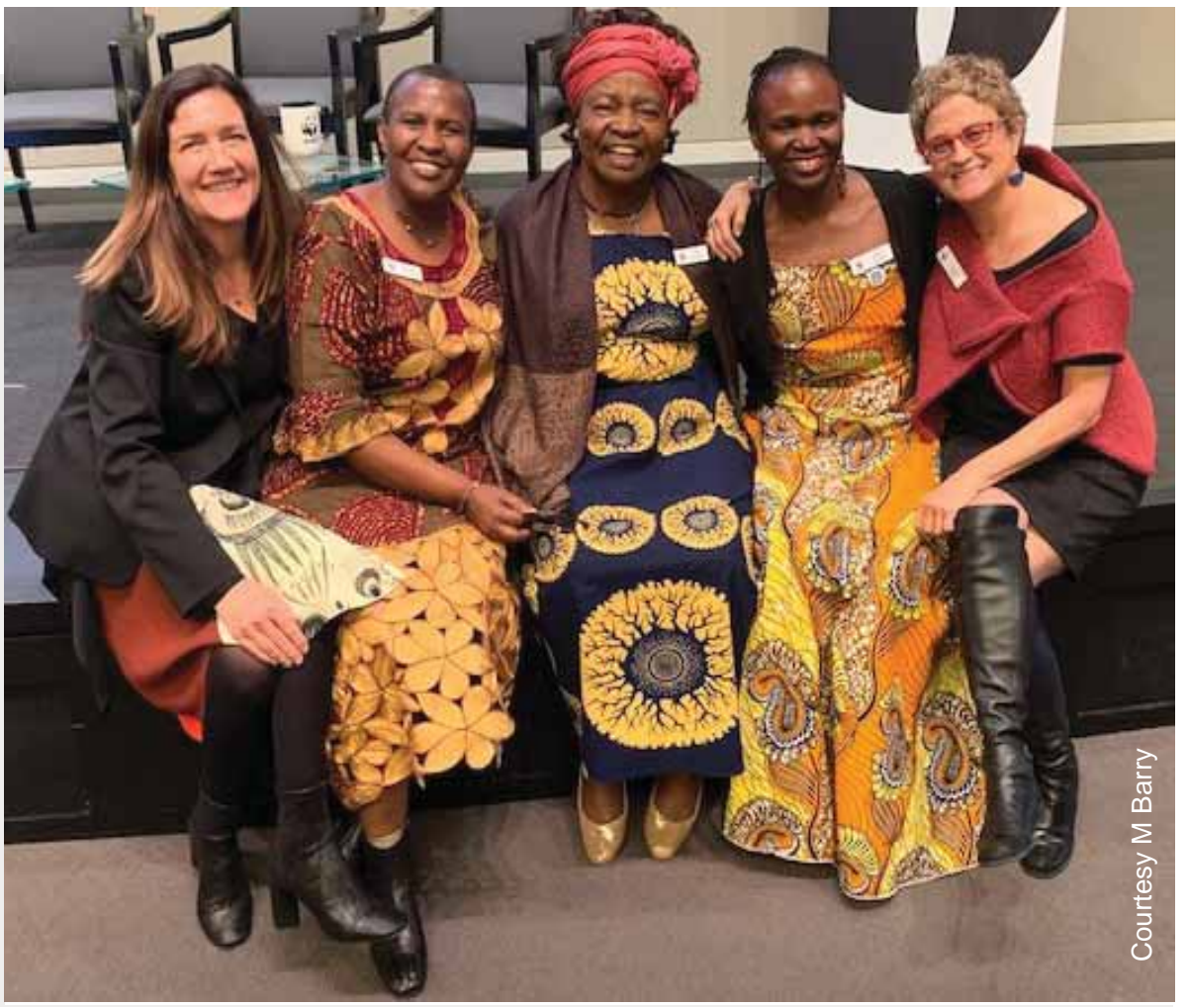

MEDICC Review: COVID-19, now a pandemic, is not the first disease to reach epidemic proportions in recent years. It's calculated that from 2011 to 2018, there were 1483 epidemic outbreaks in 172 countries. And in the Americas alone, we had Zika, dengue, yellow fever, drug-resistant malaria, pulmonary hantavirus syndrome, cholera and H1N1. Looking at the big picture, what should the world have learned that we didn't?

Michele Barry: We need to sit back and realize that we all live on one planet and disease knows no borders. Many of these recent epidemics such as Ebola, yellow fever and Zika started in conflict zones or in countries which have fragile health systems and more vulnerable populations. The concept of interdependent sovereignty needs to be acknowledged for pandemic disease. Early surveillance and healthcare system strengthening becomes the responsibility of all countries.

MEDICC Review: You've identified the climate crisis as one of the underlying triggers of emerging and re-emerging infections. Is it the game changer right now?

Michele Barry: Climate change is important. But what I feel is even more important is the fact that we are living in the Anthropocene age, the geologic age when human activities have dramatically affected our planet and its environment. So yes, there is dramatic climate change, which can affect vector distribution of these diseases, but more important in my mind are other human activities which redistribute risk such as urbanization, deforestation, global travel and the human interface and relationship with animals. The majority of epidemics-Ebola, HIV, yellow fever and even now COVID-19-have to do with relationships to animals and what we call zoonotic diseases, the spillover from animal to human beings.

MEDICC Review: Yet, in Brazil, where Zika erupted, it seems government leaders are making light of the current pandemic....and continuing with the deforestation of the Amazon, the "lungs of the world."

Michele Barry: It is no surprise that Zika hit an area in Brazil that had been deforested and urbanized in the northeast. Right before the Zika outbreak, there had been a drought and people were collecting rainwater in buckets-a perfect breeding ground for Aedes aegypti-a ferocious daybiting mosquito whose larvae can proliferate in the water of a jar cap. I also was involved in identifying the clinical presentation of a new hemorrhagic virus called Sabia from Brazil many years ago. This virus was found in 


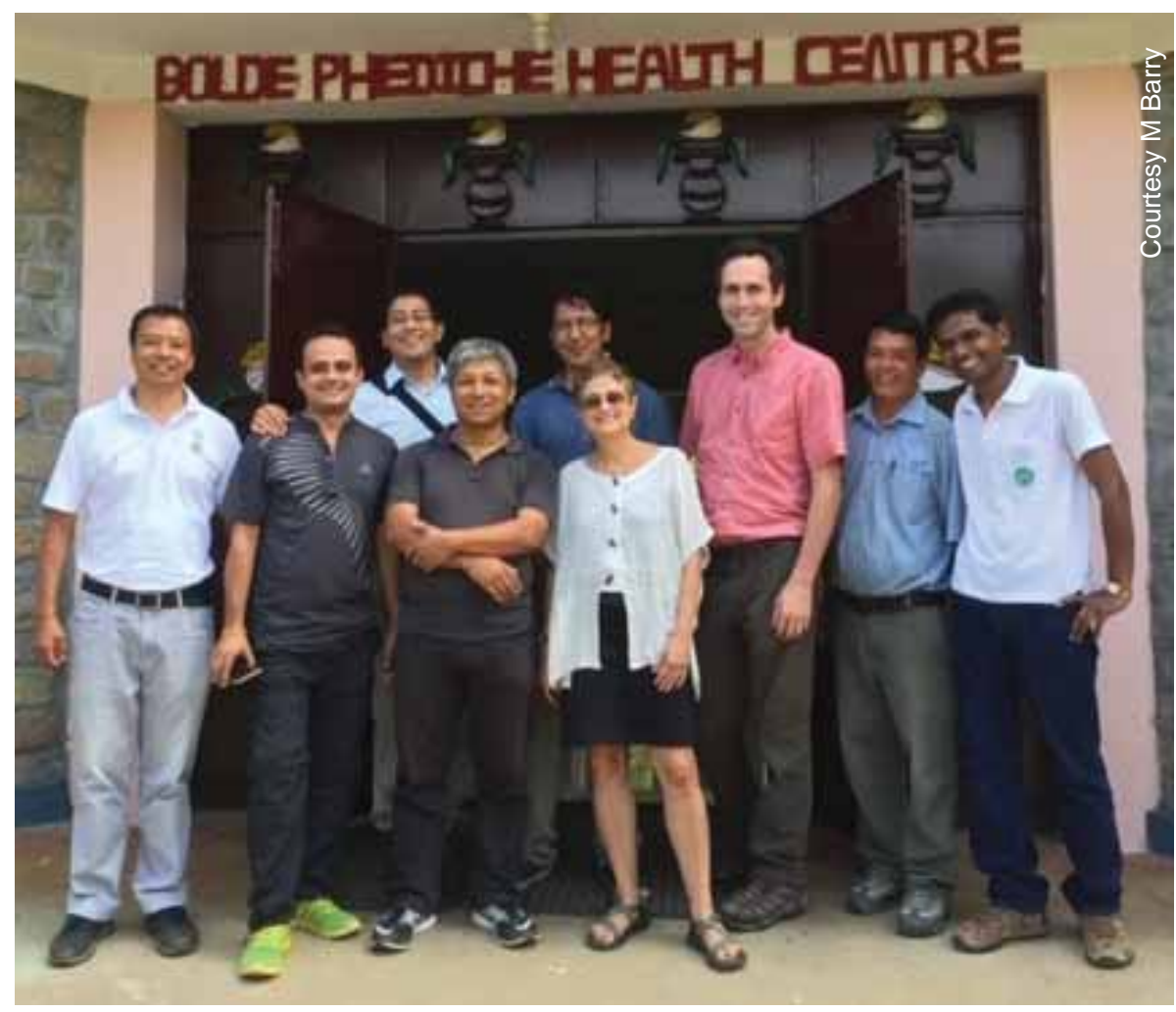

Michele Barry (c.) in Nepal working on typhoid project.

rodent excretions around deforested areas in the Amazonian area that humans had new contact with.

MEDICC Review: What can and should governments and peoples do differently, intersectorally? How can professionals and institutions in the health sector help push them to do it?

\section{We have seen how well governments do with a centralized coordinated approach to public health}

Michele Barry: First, we have seen how well governments do with a centralized coordinated approach to public health. This was emphasized by how China, Singapore, Taiwan and South Korea handled their outbreaks, although of course the world is waiting to see if there is a resurgence once restrictions are lifted. Intersectionality is key-good medicine cannot only be about high technology research and treatment geared to the individual. It also needs to model public population health by emphasizing disease prevention, behavioral changes for health and do this via leadership diversity. Precision medicine, a trendy name these days, should not only be about targeting the individual genome, but we need to precisely diagnose and understand the population "genome." Lastly, health professionals need to put their weight behind strengthening WHO's international health regulations, which set minimum standards for diagnosing and conducting surveillance for epidemic disease.

MEDICC Review: Are scientists themselves called upon to change the way they work? Have we already seen more collaboration among scientists in this pandemic?

Michele Barry: Yes. The Chinese were early to share the genome of the COVID-19 Wuhan strain. Labs around the world are turning their sequencing machines, most about the size of a desktop printer, to the task of rapidly sequencing the genomes of virus samples taken from the sick. The information is uploaded to a website called nextstrain.org that shows how the virus is migrating and splitting into similar but new subtypes. Fortunately, because it is so successful at infecting people, there appears not to be much pressure for it to mutate rapidly although we have seen slow small mutations. Another way scientists are collaborating is through journals, which are rapidly sharing information online and in preprint-far faster than business as usual; most journals are waiving any costs or fees for access to these findings.

\section{MEDICC Review: Does COVID-19 challenge} the US health system to change?

Michele Barry: One hot spot of virus can quickly overwhelm the system. But in the long run, is this a wakeup call to the US to restructure their pandemic preparedness and population health capacity? I believe strongly there should be a cabinet level position for global and population health. The US needs to rethink a medical care system that may be one of the best in the world for research and curative medicine but currently is a patchwork of decentralized public health responses that answer to no one federal branch. Even Centers for Disease Control and Prevention (CDC) needs to be invited into a state before they can help the public around an urgent issue.

MEDICC Review: Have we focused a gender lens on COVID-19, from the $70 \%$ of global health workers who are women, to the implications for health of women and families worldwide? What more can be done to bring a gender perspective into play?

Michele Barry: First, we need to get good disaggregated genderspecific data. From China we found that women did a bit better with the disease vis-a-vis survival, but we don't have a good understanding why this is. Men tend to smoke more and may have had more comorbid disease, yet women live longer so were an older population known to be at greater risk. We need to tease these factors out.

However, we do know some things to be true: intimate domestic violence rises in pandemic situations-we saw this postearthquake in New Zealand and we saw this with Ebola in western Africa. We also know that women bear the brunt of housekeeping, cooking and childcare in most societies when quarantine occurs. Pregnant women need to get antenatal and labor and delivery care that can be offered in a well-functioning health system. And lastly the majority of frontline caretakers are female nurses putting themselves at greatest risk.

On a small personal note, I would like to see more of my women colleague COVID-19 experts in the forefront as key deciders and presenters of policy, as well as being funded to do the science. $-1 /$ - 